\title{
Ramucirumab for Patients with Intermediate- Stage Hepatocellular Carcinoma and Elevated Alpha-Fetoprotein: Pooled Results from Two Phase 3 Studies (REACH and REACH-2)
}

\author{
Masatoshi Kudo $^{a}$ Richard S. Finn $^{b}$ Manabu Morimoto ${ }^{c}$ Kun-Ming Rau ${ }^{d, e}$

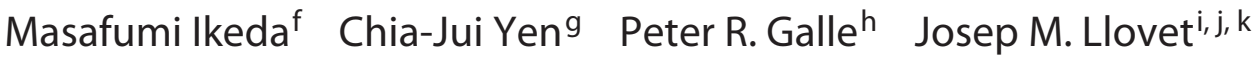 \\ Bruno Daniele ${ }^{l}$ Ho Yeong Lim ${ }^{m}$ David W. Mcllwain ${ }^{n}$ Reigetsu Yoshikawa ${ }^{\circ}$ \\ Kenichi Nakamura ${ }^{\circ}$ Kun Liang $^{\mathrm{n}}$ Chunxiao Wang $^{\mathrm{n}}$ Paolo Abada ${ }^{\mathrm{n}}$ \\ Ryan C. Widau ${ }^{\mathrm{n}}$ Andrew X. Zhup ${ }^{\mathrm{p}, \mathrm{q}}$

\begin{abstract}
a Departments of Gastroenterology and Hepatology, Kindai University, Osaka, Japan; b Geffen School of Medicine, University of California, Los Angeles, CA, USA; 'Kanagawa Cancer Center, Yokohama, Japan; ${ }^{\mathrm{d} C h a n g}$ Gung Memorial

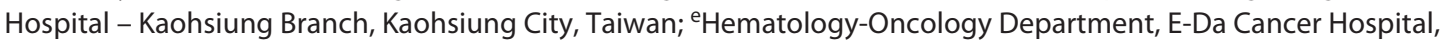
Kaohsiung, Taiwan; 'Department of Hepatobiliary and Pancreatic Oncology, National Cancer Center Hospital East, Kashiwa, Japan; ${ }^{9}$ Department of Internal Medicine, College of Medicine, National Cheng Kung University, Tainan, Taiwan; ' Department of Internal Medicine, Mainz University Medical Center, Mainz, Germany; 'Mount Sinai Liver Cancer Program, Department of Liver Diseases, Icahn School of Medicine at Mount Sinai, New York, NY, USA; 'ं Liver Cancer Translational Research Laboratory, Institut d'Investigacions Biomèdiques August Pi i Sunyer (IDIBAPS)Hospital Clínic, Universitat de Barcelona, Barcelona, Spain; ${ }^{k}$ nstitució Catalana de Recerca i Estudis Avançats (ICREA), Barcelona, Spain; 'Ospedale del Mare, Naples, Italy; ${ }^{\mathrm{m} S a m s u n g}$ Medical Center, Sungkyunkwan University, Seoul, South Korea; "Eli Lilly and Company, Indianapolis, IN, USA; ${ }^{\circ}$ Eli Lilly Japan K.K., Kobe, Japan; ${ }^{P}$ Massachusetts General Hospital Cancer Center, Boston, MA, USA; ${ }^{9}$ Jiahui International Cancer Center, Jiahui Health, Shanghai, China
\end{abstract}

\section{Keywords}

Ramucirumab · Barcelona clinic liver cancer stage ·

a-fetoprotein

\begin{abstract}
Background: Intermediate-stage hepatocellular carcinoma (HCC), as defined by Barcelona Clinic Liver Cancer (BCLC) stage $B$, is heterogeneous in terms of liver function and tumor burden. REACH and REACH-2 investigated ramucirumab in patients with HCC after prior sorafenib, with REACH-2 enrolling only patients with baseline a-fetoprotein (AFP)
\end{abstract}

$\geq 400 \mathrm{ng} / \mathrm{mL}$. An exploratory analysis of outcomes by BCLC stage was performed. Methods: A pooled meta-analysis of independent patient data (stratified by study) from REACH (AFP $\geq 400 \mathrm{ng} / \mathrm{mL}$ ) and REACH-2 was performed. All patients had Child-Pugh A, Eastern Cooperative Oncology Group performance status $0-1$, prior sorafenib treatment, and either HCC BCLC stage B (refractory/not amenable to locoregional therapy) or BCLC stage C. Patients were randomized to ramucirumab $8 \mathrm{mg} / \mathrm{kg}$ or placebo every 2 weeks. Median overall survival (OS) and progression-free survival were estimated by the Kaplan-Meier method. Treatment effects in BCLC stage $B$ and $C$ were evaluated by Cox proportional-hazards
(C) 2021 The Author(s)

Published by S. Karger AG, Basel

This is an Open Access article licensed under the Creative Commons Attribution-NonCommercial-4.0 International License (CC BY-NC) (http://www.karger.com/Services/OpenAccessLicense), applicable to the online version of the article only. Usage and distribution for commercial purposes requires written permission.
Correspondence to:

Masatoshi Kudo, m-kudo@med.kindai.ac.jp 
model; prognosis of BCLC staging for OS was evaluated by multivariate Cox proportional-hazards model. Tumor responses were evaluated according to Response Evaluation in Solid Tumors v1.1. Liver function was assessed with albumin-bilirubin score. Results: Baseline characteristics were generally balanced between treatment arms in each BCLC stage. BCLC staging trended as an independent prognostic factor for OS (B vs. C; hazard ratio [HR] 0.756 [95\% Cl 0.5461.046]). Consistent treatment benefit was observed for ramucirumab versus placebo across BCLC stages. Median OS for ramucirumab versus placebo was 13.7 versus 8.2 months; HR (95\%): 0.43 (0.23-0.83) and 7.7 versus 4.8 months; HR (95\%): 0.72 (0.59-0.89) for BCLC stage B and C, respectively. Adverse events (AEs) were consistent with observations from both studies; hypertension was the most frequent grade $\geq 3 \mathrm{AE}$. Liver function was preserved throughout the study and similar between treatment arms in both BCLC stages. Conclusions: Ramucirumab provided a better survival benefit irrespective of BCLC stage and was well tolerated without compromising liver function during treatment.

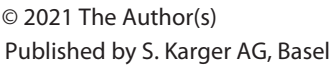

\section{Background}

The Barcelona Clinic Liver Cancer (BCLC) staging system categorizes patients into 4 main prognostic groups (stages A, B, C, and D) based on tumor burden, severity of liver disease, and Eastern Cooperative Oncology Group performance status (ECOG PS) [1-3]. Intermediate-stage hepatocellular carcinoma (HCC) is classified as BCLC stage $\mathrm{B}$ and is a heterogeneous disease in terms of liver function and tumor burden $[4,5]$. BCLC stage $B$ includes all patients having a Child-Pugh score of A or B with an ECOG PS 0, who also have a single nodule $>5 \mathrm{~cm}$, or multiple nodules $>3$ in number, or at least one of these $>3 \mathrm{~cm}$, without macrovascular invasion and extrahepatic spread [6]. Transarterial chemoembolization (TACE) is the recommended first-line treatment in most clinical practice guidelines with over half of all patients with intermediatestage HCC treated with this procedure [7-9]. Due to population heterogeneity, however, the treatment benefits observed with TACE have been variable with a high rate of tumor recurrence. There is a wide gap between clinical practice guidelines and applied clinical practice with regards to intermediate HCC [10-12]. Recently, lenvatinib has been shown to benefit patients with intermediate HCC when compared with TACE in the unresectable setting [5]. While intermediate HCC patients may initially be treated with locoregional therapies, nearly all patients experience disease progression and require systemic therapy [13]. Actually, evidence of TACE efficacy was established by meta-analysis of several randomized controlled trials comparing TACE with no therapy (best supportive care) when systemic therapy was virtually not existing [14]. In other words, there is no evidence that TACE or systemic therapy is better in intermediate-stage HCC with high tumor burden.

A number of systemic treatments are currently being investigated and/or are approved for the treatment of HCC [15]. Tyrosine kinase inhibitors (e.g., sorafenib, lenvatinib, regorafenib, and cabozantinib) have shown positive survival benefits for patients with intermediate and advanced HCC [16-20]. Checkpoint inhibitors such as atezolizumab (in combination with bevacizumab), nivolumab, and pembrolizumab have also shown efficacy in these patient populations [21-24]. Recently, an antiangiogenic agent, ramucirumab, has demonstrated improved overall survival (OS) in patients with advanced HCC and elevated $\alpha$-fetoprotein (AFP) [25-27].

REACH (Clinicaltrials.gov Identifier NCT01140347) andREACH-2 (Clinicaltrials.govIdentifierNCT02435433) were randomized, placebo-controlled, phase 3, clinical trials that investigated ramucirumab (IgG1 vascular endothelial growth factor receptor 2 antagonist) in patients with HCC who progressed on or were intolerant to sorafenib $[25,26]$. REACH-2 only enrolled patients with baseline AFP levels $\geq 400 \mathrm{ng} / \mathrm{mL}$ [25]. In both clinical trials, patients with BCLC stage C disease or BCLC stage B disease not amenable or refractory to locoregional therapy were included $[25,26]$. Treatment with ramucirumab provided survival benefits in REACH-2 and in those patients with a baseline AFP $\geq 400 \mathrm{ng} / \mathrm{mL}$ in REACH $[25$, $26]$. Here, we show the results of an exploratory analysis conducted to investigate the efficacy and safety of ramucirumab in advanced HCC patients with elevated AFP from REACH-2 and REACH by BCLC stage.

\section{Materials and Methods}

REACH and REACH-2 were randomized, placebo-controlled, double-blind, multicenter, phase 3 trials (Fig. 1). Patients either received intravenous ramucirumab $(8 \mathrm{mg} / \mathrm{kg})$ or placebo every 2 weeks (REACH randomized 1:1; REACH-2 randomized 2:1), plus best supportive care, until disease progression, unacceptable toxicity, or death $[25,26]$. An individual patient data meta-analysis was performed on REACH-2 patients [25] and REACH patients with AFP $\geq 400 \mathrm{ng} / \mathrm{mL}$ [21] to investigate the efficacy and safety of ramucirumab in advanced HCC patients by BCLC stage: pooled results were stratified by study. Pooling the patient-level data increased the patient population and allowed for a more precise es- 
REACH

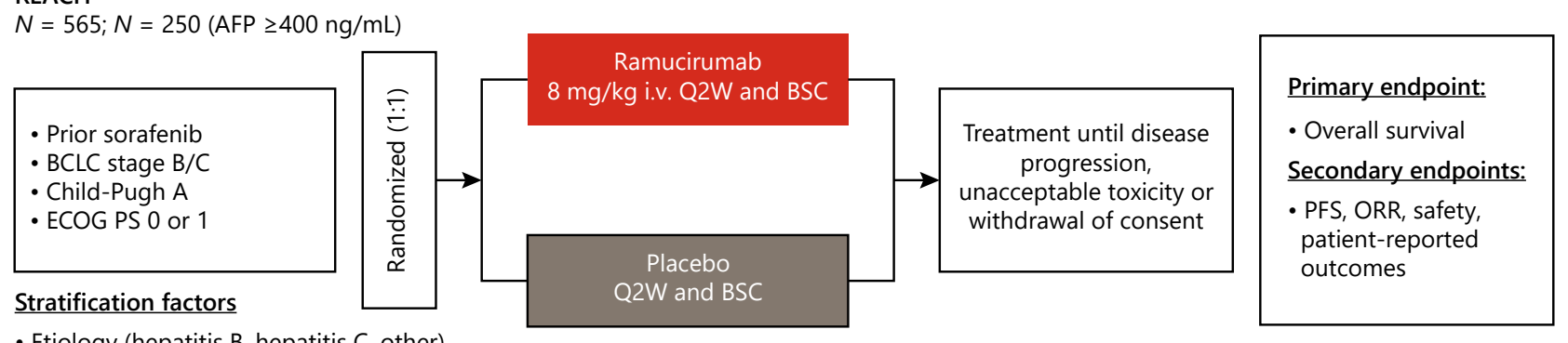

- Etiology (hepatitis B, hepatitis C, other)

- Geographic regions

REACH-2

$N=292$

- Prior sorafenib
- BCLC stage B/C
- Child-Pugh A
- ECOG PS 0 or 1
- Baseline AFP $\geq 400 \mathrm{ng} / \mathrm{mL}$

Stratification factors

Macrovascular invasic

ECOG PS (0 vs. 1)

Geographic regions
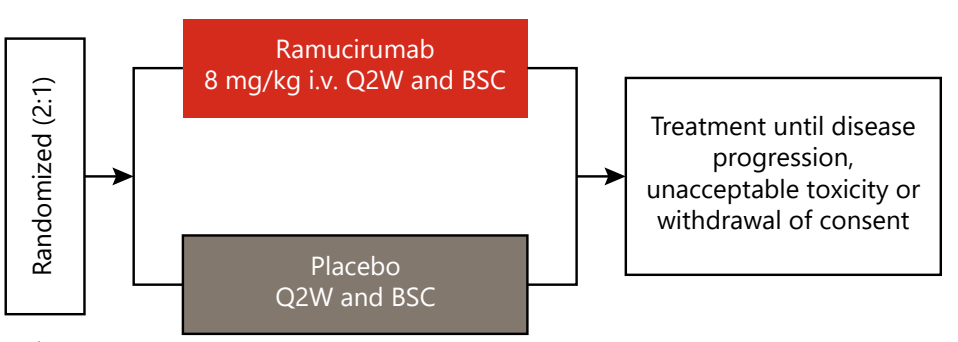

Primary endpoint:

- Overall survival

Secondary endpoints:

- PFS, ORR, safety, patient-reported outcomes

Fig. 1. REACH and REACH-2 clinical study designs. AFP, $\alpha$-fetoprotein; BCLC, Barcelona Clinic Liver Cancer; BSC, best supportive care; ECOG PS, Eastern Cooperative Oncology Group performance status; i.v., intravenous; $N$, number of participants; ORR, objective response rate; PFS, progression-free survival; Q2W, every 2 weeks. ClinicalTrials.gov identifier: REACH: NCT01140347, REACH-2: NCT02435433.

timation of treatment effect in subgroup analyses. Outcomes of patients in the pooled population were reported by baseline BCLC stage B or C. BCLC staging for this analysis was derived based on individual patient data entered in the electronic case report form prior to the start of second-line therapy.

Both OS and progression-free survival (PFS) were assessed using the Kaplan-Meier method, and hazard ratios (HRs) were assessed by univariate and multivariate Cox proportional-hazards models accounting for covariates of treatment, BCLC stage, and baseline AFP. Objective response rate, disease control rate, and safety were reported by BCLC stage. All response assessments were done locally by investigators according to Response Evaluation in Solid Tumors version 1.1 and adverse events (AEs) were graded according to the Common Terminology Criteria for AEs version 4.0. Laboratory monitoring, including serum albumin and bilirubin levels, were measured within 14 days prior to randomization and before administration of study drug at each cycle (every 14 days) per the protocol $[25,26]$. The albumin-bilirubin (ALBI) grade was used as an assessment of overall liver function $[28,29]$. The ALBI linear predictor, ALBI score, was determined using the following formula: ALBI linear predictor $=\left(\log _{10}\right.$ bilirubin $\left.\times 0.66\right)+$ (albumin $\times-0.085$ ), where bilirubin is in $\mu \mathrm{mol} / \mathrm{L}$ and albumin in $\mathrm{g} / \mathrm{L}$. Patients were categorized into the ALBI grades by applying cutoff to the linear predictor: grade $1: \leq-2.60$; grade 2 : $>-2.60$ to $\leq$ -1.39 ; and grade $3:>-1.39[28,29]$.

Ramucirumab for Intermediate-Stage

Hepatocellular Carcinoma

\section{Results}

Data from 52 patients (ramucirumab, $N=30$ and placebo, $N=22$ ) with BCLC stage B disease and data from 490 patients (ramucirumab $N=286$; placebo $N=204$ ) with BCLC stage $\mathrm{C}$ disease were analyzed (Table 1). Baseline patient and disease characteristics were generally well-balanced across treatment arms. Region 3 (Japan) had a higher percentage of patients with BCLC stage B, while patients with BCLC stage $C$ were more common in Region 2 (Asia excluding Japan). Additionally, patients with BCLC stage $C$ had higher median levels of AFP (ramucirumab arm: 4,472 ng/mL and placebo arm: 4,375 $\mathrm{ng} / \mathrm{mL}$ ) than patients with BCLC stage B disease (ramucirumab arm: 1,817 ng/mL and placebo arm: 2,958 ng/ $\mathrm{mL})$. In the overall study population, more than half of all patients with BCLC stage B or BCLC stage C disease had undergone TACE prior to systemic treatment.

To investigate what factors were significantly associated with survival, univariate and multivariate analyses of OS adjusted for baseline BCLC stage were performed. BCLC 
Table 1. Baseline demographics and disease characteristics

\begin{tabular}{|c|c|c|c|c|}
\hline$n(\%)$ except where indicated & \multicolumn{2}{|l|}{ BCLC stage B } & \multicolumn{2}{|l|}{ BCLC stage C } \\
\hline Gender, male & $27(90)$ & $19(86)$ & $219(77)$ & $170(83)$ \\
\hline Age, years, median & 65 & 65 & 63 & 62 \\
\hline ECOG PS 0 & $30(100)$ & $22(100)$ & $143(50)$ & $96(47)$ \\
\hline Region 2 (Asia excluding Japan) & $3(10)$ & $2(9)$ & $98(34)$ & $76(37)$ \\
\hline Region 3 (Japan) & $13(43)$ & $8(36)$ & $48(17)$ & $32(16)$ \\
\hline Child-pugh score A-5 & $22(73)$ & $12(55)$ & $168(59)$ & $123(60)$ \\
\hline Prior TACE & $22(73)$ & $11(50)$ & $157(55)$ & $112(55)$ \\
\hline \multicolumn{5}{|l|}{ Number of prior TACE } \\
\hline 0 & $8(27)$ & $11(50)$ & $129(45)$ & $92(45)$ \\
\hline Extrahepatic spread present & 0 & 0 & $226(79)$ & $171(84)$ \\
\hline Median duration of prior sorafenib, months & 4.8 & 5.2 & 3.6 & 3.9 \\
\hline Median AFP (IQR), ng/mL & 1,817 & 2,958 & 4,472 & 4,375 \\
\hline & $(1,000-6,425)$ & $(1,591-12,317)$ & $(1,283-24,494)$ & $(1,174-23,120)$ \\
\hline \multicolumn{5}{|l|}{ ALBI grade } \\
\hline 1 & $15(50)$ & $9(41)$ & $121(42)$ & $86(42)$ \\
\hline 2 & $15(50)$ & $13(59)$ & $161(56)$ & $107(53)$ \\
\hline 3 & 0 & 0 & $1(0.3)$ & $6(3)$ \\
\hline \multicolumn{5}{|l|}{ Etiology of liver disease } \\
\hline Hepatitis B virus & $12(40)$ & $6(27)$ & $112(39)$ & $96(47)$ \\
\hline Hepatitis $\mathrm{C}$ virus & $10(33)$ & $10(46)$ & $73(26)$ & $46(23)$ \\
\hline
\end{tabular}

AFP, a-fetoprotein; ALBI, albumin-bilirubin; BCLC, Barcelona Clinic Liver Cancer; ECOG PS, Eastern Cooperative Oncology Group performance status; IQR, interquartile range; TACE, transarterial chemoembolization.

stage was prognostic for OS in the univariate analysis (stage $B$ vs. stage C; HR 0.68, 95\% CI 0.49-0.94; $p$ value 0.02 ). When we adjusted for treatment and baseline AFP in the multivariate analysis, it did not reach statistical significance (stage B vs. stage C; HR 0.76, 95\% CI $0.55-1.05$; $p$ value 0.0917 ) (see online suppl. Table 1; for all online suppl. material, see www.karger.com/doi/10.1159/000516605).

Ramucirumab provided a benefit to OS and PFS irrespective of BCLC stage (Fig. 2a, b). No significant difference in treatment effect by BCLC subgroup was detected (OS interaction $p$ value $=0.273$; PFS interaction $p$ value $=$ 0.432 ), indicating a comparable benefit with ramucirum$\mathrm{ab}$ in patients with BCLC stage $\mathrm{B}$ and $\mathrm{C}$ disease. For BCLC stage $B$, the median OS for the ramucirumab arm versus placebo was 13.7 versus 8.2 months (HR $0.43,95 \%$ CI
$0.23-0.83$ ) and the median PFS was 4.2 versus 2.8 months (HR 0.33, 95\% CI 0.17-0.64). For BCLC stage C, the median OS for the ramucirumab arm versus placebo was 7.7 versus 4.8 months (HR $0.72,95 \%$ CI $0.59-0.89$ ) and the median PFS was 2.8 versus 1.5 months (HR 0.60 , 95\% CI 0.49-0.74).

Differences in post-discontinuation therapies (PDTs) (online suppl. Table 2) and AFP (Table 1) were noted in BCLC stage B; however, the OS benefit persisted after adjusting for AFP levels (online suppl. Fig. 1) and PDTs (online suppl. Fig. 2). A higher proportion of intermediatestage patients in the ramucirumab arm experienced an objective response $(n=5,17 \%)$ and disease control $(n=$ $24,80 \%)$ than patients with advanced-stage disease (4\% and $54 \%$, respectively) (Table 2). 
a

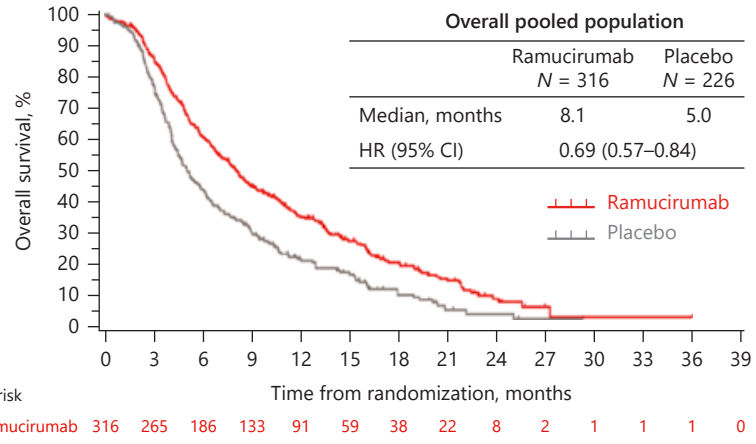

$\begin{array}{lcccccccccccccc}\text { Ramucirumab } & 316 & 265 & 186 & 133 & 91 & 59 & 38 & 22 & 8 & 2 & 1 & 1 & 1 & 0 \\ \text { Placebo } & 226 & 166 & 94 & 64 & 37 & 26 & 12 & 5 & 3 & 1 & 0 & 0 & 0 & 0\end{array}$

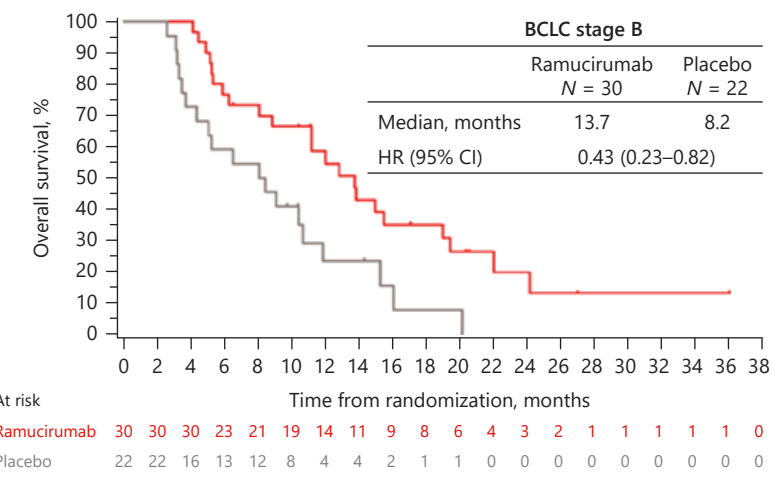

$\begin{array}{lllllllllllllllllllll}\text { Placebo } & 22 & 22 & 16 & 13 & 12 & 8 & 4 & 4 & 2 & 1 & 1 & 0 & 0 & 0 & 0 & 0 & 0 & 0 & 0 & 0\end{array}$

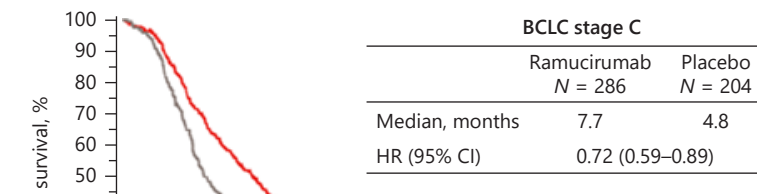

At risk

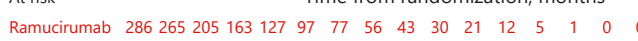

Placebo $\quad 204 \begin{array}{llllllllllllllll}177 & 120 & 81 & 62 & 46 & 33 & 27 & 18 & 11 & 8 & 4 & 3 & 1 & 1 & 0\end{array}$

ठั

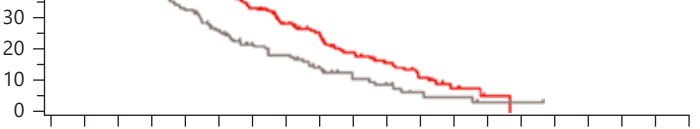

$\begin{array}{llllllllllllllllllll}0 & 2 & 4 & 6 & 8 & 10 & 12 & 14 & 16 & 18 & 20 & 22 & 24 & 26 & 28 & 30 & 32 & 34 & 36 & 38\end{array}$ Time from randomization, months b
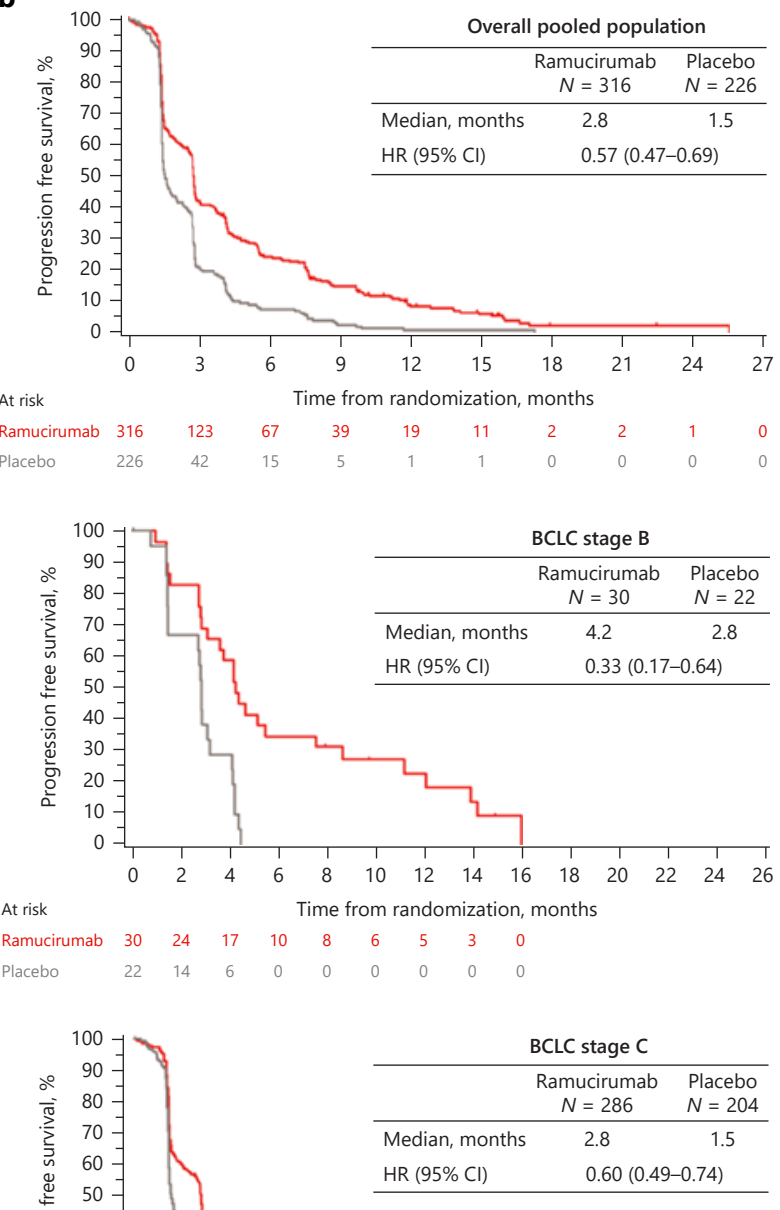

Fig. 2. OS (a) and PFS (b) by baseline BCLC stage. Kaplan-Meier analysis for OS and PFS shown by treatment arm (ramucirumab [red] vs. placebo [grey]) for pooled REACH and REACH-2 patients by BCLC stage; BCLC B $(N=52)$ and BCLC C $(N=487)$. A Wald test of treatment-by-subgroup interaction from stratified Cox model was used, stratified by study identification. BCLC, Barcelona Clinic Liver Cancer (staging categories); CI, confidence interval; HR, hazard ratio; $\mathrm{N}$, number of patients in intent-to-treat population; OS, overall survival; PFS, progression-free survival.

Compared with baseline values, an incremental increase in the mean ALBI score was noted in both ramucirumab- and placebo-treated patients at the end of study treatment (Fig. 3). This incremental increase was similar in both treatment arms and BCLC stages and was not sig- nificant enough for a worsening of the ALBI grade. Treatment-emergent AEs (TEAEs) were consistent with those found in the intent-to-treat population of REACH and REACH-2. Hypertension was the most frequent grade $\geq 3$ TEAE (Table 3 ) and no new safety signals were detected. 
Table 2. Best overall response (RECIST v 1.1) by baseline BCLC stage

\begin{tabular}{llllll}
\hline$n(\%)$ & BCLC stage $B$ & & BCLC stage C & \\
\cline { 2 - 3 } & ramucirumab, $N=30$ & placebo, $N=22$ & & ramucirumab, $N=286$ & placebo, $N=204$ \\
\hline ORR & $5(17)$ & $1(5)$ & $12(4)$ & $1(1)$ \\
DCR & $24(80)$ & $13(59)$ & $154(54)$ & $71(35)$ \\
\hline
\end{tabular}

All response assessments were done locally by investigators according to RECIST (version 1.1). BCLC, Barcelona Liver Cancer Clinic; RECIST, Response Evaluation in Solid Tumors; ORR, objective response rate; DCR, disease control rate.

Fig. 3. Effect of study treatment on ALBI score/grade by baseline BCLC stage. ALBI linear predictor was used as a measure of liver function over the course of the treatment cycle. The numbers of patients in each group at baseline were RAM BCLC B $(N=30$, red $)$; PL BCLC B $(N=22$, grey); RAM BCLC C $(N=283$, blue $)$; and PL BCLC C $(N=199$, green). Patients were categorized into the ALBI grades using cutoffs: Grade 1: $\leq-2.60$; Grade 2: $>-2.60$ to $\leq$ -1.39 ; and Grade 3: $>-1.39$. ALBI, albumin-bilirubin; BCLC, Barcelona Clinic Liver Cancer (staging categories); BS, baseline; C 1-9, cycle 1-9; EOT, end of treatment; PL, placebo; RAM, ramucirumab.

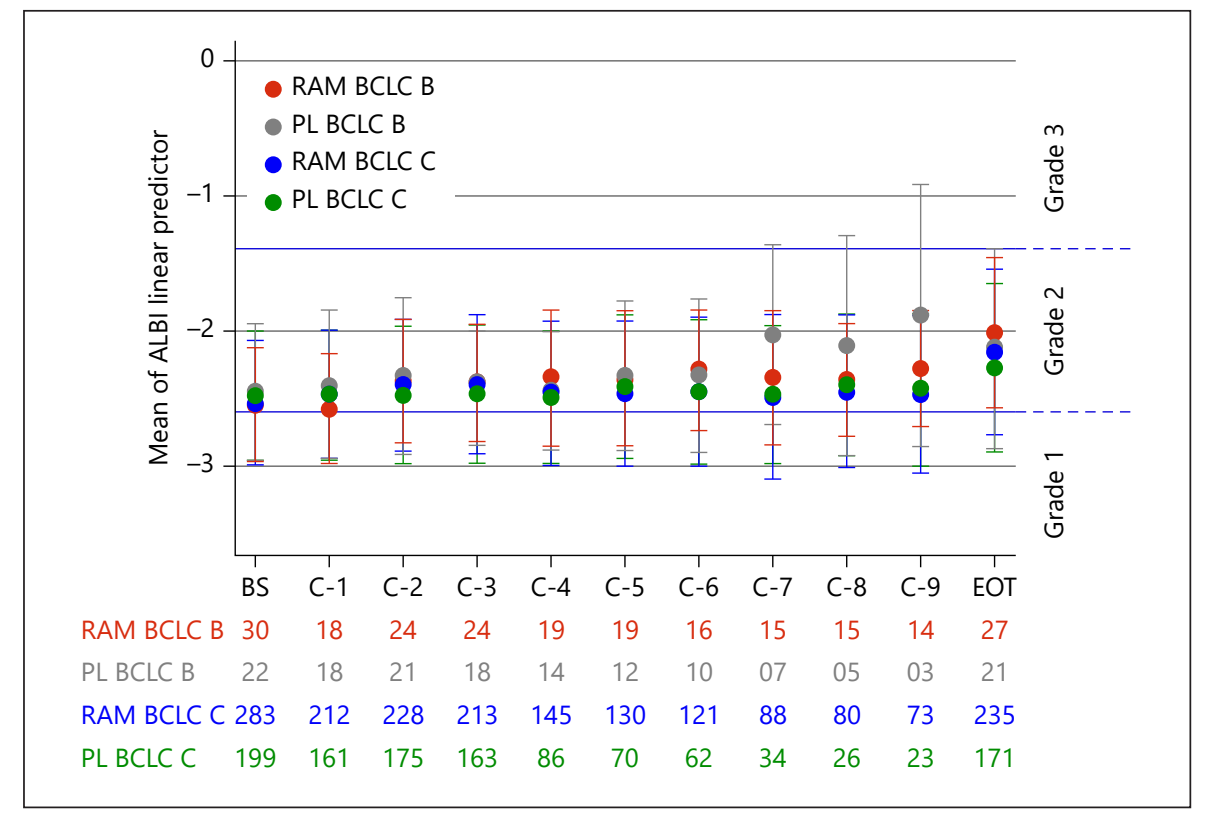

\section{Discussion}

In this exploratory, post-hoc analysis, we investigated the efficacy and safety of ramucirumab by BCLC stage in patients from REACH $(\mathrm{AFP} \geq 400 \mathrm{ng} / \mathrm{mL})$ and REACH-2. We found that ramucirumab demonstrated improved survival outcomes over placebo for patients with intermediate and advanced-stage HCC. The survival benefit of ramucirumab versus placebo observed in patients with BCLC stage B was numerically greater than in those with BCLC stage C (median delta of +5.5 vs. +2.9 months). This benefit in OS remained after accounting for differences in baseline AFP levels and PDT. The baseline median AFP levels of the BCLC stage C patients were much higher than those of the BCLC stage $\mathrm{B}$ patients, consistent with other studies that have demonstrated that AFP levels are known to correlate with HCC subclasses and elevated levels are associated with poor survival outcomes [30].
The higher percentage of patients in the ramucirumab arm with BCLC stage B versus BCLC stage $C$ who received PDT ( $40 \%$ vs. $29 \%$, respectively) may suggest that earlier introduction of molecularly targeted drugs, including ramucirumab, at the intermediate stage may lead to increased opportunities for PDT and longer survival $[5,31]$.

The BCLC staging system is well-known for establishing treatment strategies and potential prognoses associated with each of the 4 main stages [2]. In our study, BCLC stage was prognostic for OS in the univariate analysis (stage B vs. stage C; HR 0.68, 95\% CI 0.49-0.94) but did not reach significance when we adjusted for treatment and baseline AFP in the multivariate analysis (stage B vs. stage C; HR 0.76, 95\% CI 0.55-1.05). The smaller population size of patients with BCLC stage B disease from our pooled population likely contributed to the lack of statistical significance. 
Table 3. TEAEs by baseline BCLC stage

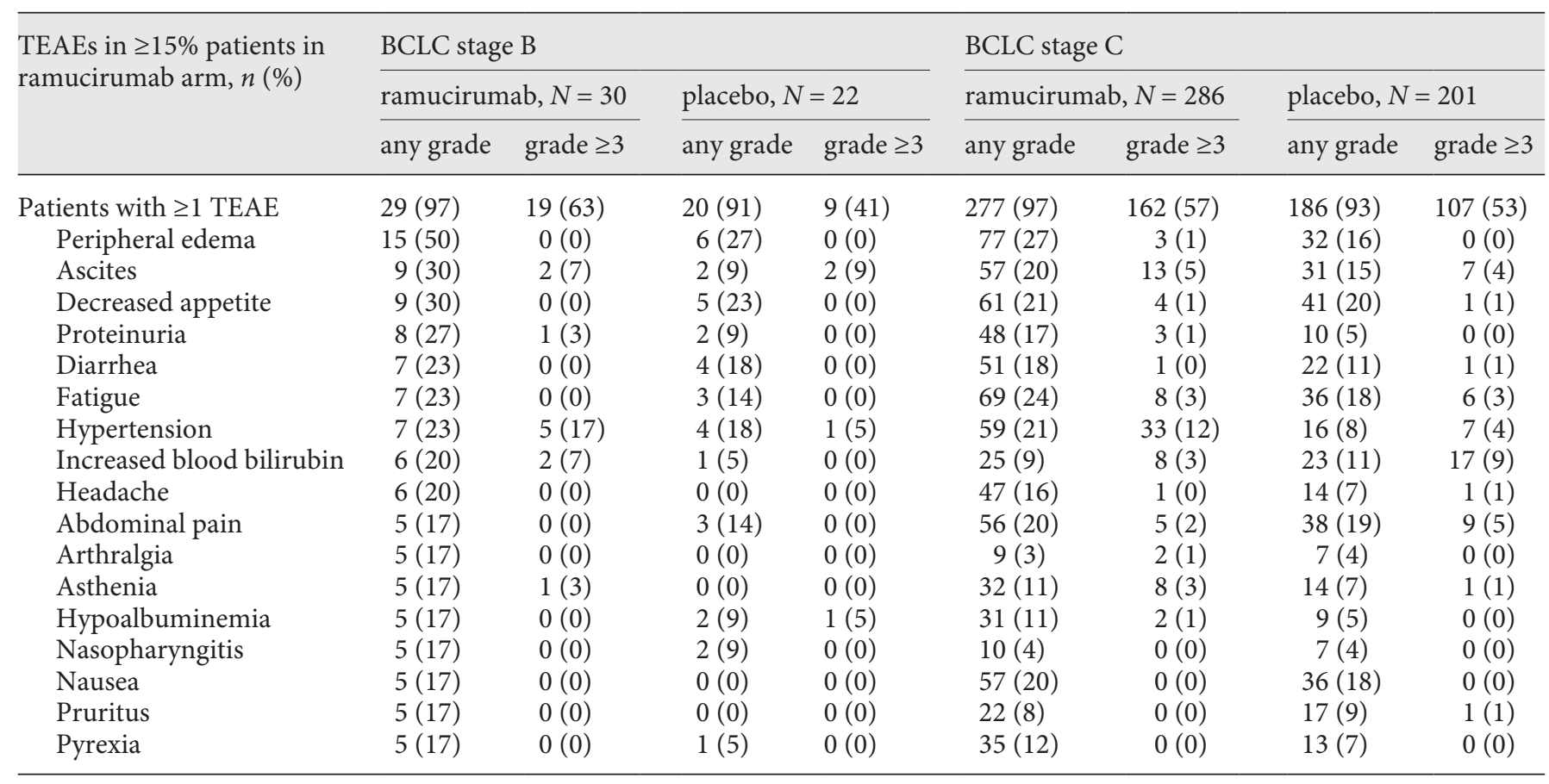

AEs were graded according to the CTCAE (version 4.0). AE, adverse event; BCLC, Barcelona Clinic Liver Cancer; CTCAE, Common Terminology Criteria for Adverse Events; TEAE, treatment-emergent adverse event.

No new safety signals were detected during this analysis when compared with the intent-to-treat population, with hypertension being the most prevalent grade $\geq 3$ TEAE. Liver toxicity was minimal in both treatment arms. Mean ALBI score measured at baseline through the end of treatment, demonstrated baseline liver function was preserved throughout the study and was similar in each treatment arm for patients with BCLC stage B and C disease. Although an incremental increase in the mean ALBI score was noted in both ramucirumab and placebo treatment arms at the end of study treatment, it was not significant enough to increase the ALBI grade and likely represented the negative effects of disease progression.

Our study supports the second-line use of ramucirum$\mathrm{ab}$ in the treatment of patients with HCC, AFP $\geq 400 \mathrm{ng} /$ $\mathrm{mL}$, and either BCLC stage $\mathrm{B}$ who are refractory or not eligible for TACE, or BCLC stage C disease. Guidelines based on the BCLC staging system for intermediate or advanced HCC recommend TACE or systemic therapy for unresectable disease $[8,12,32,33]$. However, intermediate-stage HCC is a heterogeneous disease in terms of liver function and tumor burden and not all patients with intermediate-stage disease will benefit from TACE $[3,32$, $34,35]$. Due to this heterogeneity and the wide range of benefits observed from TACE, several groups have proposed subclassification systems for intermediate-stage HCC that allocate additional treatment options by subclass [32, 36-38]. While there have been attempts to prospectively or retrospectively validate some of these subclassification systems, none of them have gained worldwide consensus.

In our overall study population, just over half of all patients with BCLC stage B or C disease had undergone TACE prior to systemic treatment, with $14 \%$ of those patients undergoing the procedure 3 or more times. Repeated TACE utilization increases the risk for complications and has been shown to result in liver ischemia/necrosis, leading to a faster deterioration of liver function $[5,8,33$, 39-44]. Additional investigation is needed to fully discern the most appropriate treatment options in the unresectable and second-line setting that will best benefit patients with intermediate-stage HCC. For patients with intermediate-stage or advanced HCC who were unsuitable for TACE, systemic therapy with sorafenib has been the preferred option; however, lenvatinib has demonstrated noninferiority to sorafenib in the unresectable setting and may now represent an additional option. Additionally, treatment with lenvatinib recently demonstrated 
a survival benefit and preserved liver function when compared with TACE in patients with unresectable, intermediate-stage HCC with high tumor burden [5, 31].

For patients with HCC who are intolerant to or progress on sorafenib, regorafenib [18], cabozantinib [20], and ramucirumab (for patients with AFP $\geq 400$ ) [25] have been approved for second-line therapy based on large, randomized, phase 3 trials. The PD- 1 antibody, nivolumab [21], either alone or in combination with ipilimumab [45], has been granted accelerated approval in the USA based on a phase $1 / 2$ study, and outcomes of confirmatory trial of combination therapy are currently awaited. Another PD-1 antibody, pembrolizumab, has also been approved as a second-line treatment option after sorafenib based on a phase $1 / 2$ study [46]. However, both nivolum$\mathrm{ab}$ and pembrolizumab failed to show their survival benefit over comparators (sorafenib or placebo) in the phase 3 CheckMate 459 [47] and KEYNOTE-240 trials [24].

While several second-line agents are now available for patients with BCLC stage B and C disease, published reports of outcomes by BCLC stage are limited. To the best of our knowledge, no separate subgroup analyses by BCLC stage have been reported with cabozantinib in CELESTIAL [48], regorafenib in RESORCE [49], nivolumab monotherapy in CHECKMATE-040 [21], or ipilimumab + nivolumab in CHECKMATE-040 [45], nor is this data available in the primary publications. The magnitude of survival benefit was not equivalent across BCLC stage with single-agent pembrolizumab. In the KEYNOTE-240 trial, a trend in improved OS was reported in pembrolizumab-treated patients with BCLC stage C disease (HR: 0.77, 95\% CI: 0.58-1.01) with less clear activity in patients with BCLC stage B disease (HR: 0.93, 95\% CI: 0.51-1.68) [50]. Despite not being powered for subgroup analysis, these results in combination with REACH/REACH-2 data, suggest BCLC staging can affect efficacy outcomes for different systemic therapies and could be explored in future studies. One ongoing phase 4 study is assessing the efficacy and safety of various doses of cabozantinib in patients with HCC BCLC stage B who failed or are not eligible for TACE, or BCLC stage $\mathrm{C}$ who have already received 1 to 2 systemic therapies, one of which was sorafenib (CLERANCE; ClinicalTrials.gov NCT03963206). In Japan, the prospective, observational study PRISM is ongoing to explore the real-world evidence of second- or thirdline treatment in BCLC B and C stage HCC patients (UMIN ID 000040488).

A limitation of this study is the considerably smaller population of BCLC B patients compared with the BCLC $\mathrm{C}$ patients. This was likely due to the inclusion criteria of the REACH and REACH-2 studies that specified patients with BCLC stage C disease or BCLC stage B disease not amenable or refractory to locoregional therapy. Additionally, only patients with prior sorafenib treatment were included in the analysis. Additional studies are needed to determine the efficacy of second-line treatment with ramucirumab subsequent to first-line interventions other than sorafenib, and this is currently under investigation in an expansion cohort of the REACH-2 study (Clinicaltrials.gov Identifier NCT02435433) [51]. Acknowledging limitations of sample size, ramucirumab provided a survival benefit for patients with advanced HCC and elevated AFP $(\geq 400 \mathrm{ng} / \mathrm{mL})$ and did not alter liver function compared to placebo irrespective of BCLC stage.

\section{Acknowledgements}

We thank the patients and their caregivers for participating in these trials; we also thank the investigators and their support staff who generously participated in this work. Thank you to Laura Ramsey and Louise McGrath of Eli Lilly and Company for editorial and process support of this manuscript. Eli Lilly and Company contracted with Syneos Health for writing support provided by Andrea D. Humphries, PhD, and editing support provided by Antonia Baldo and Angela C. Lorio, ELS.

\section{Statement of Ethics}

REACH (NCT01140347) and REACH-2 (NCT02435433) both complied with the Declaration of Helsinki, the International Conference on Harmonization Guidelines for Good Clinical Practice, and applicable local regulations. Ethics Committees at all participating centers approved the protocol, and all patients provided written informed consent $[25,26]$.

\section{Conflict of Interest Statement}

M. Kudo is the Editor-in-Chief of Liver Cancer and reports grant and personal fees from Eisai and EA Pharma; grants from Gilead Sciences, Taiho, Sumitomo Dainippon Pharma, Takeda, Otsuka, and Abbvie; and personal fees from Roche, Ono, Eli Lilly and Company, BMS, and Bayer. R. Finn is an Editorial Board Member of Liver Cancer and reports personal fees from AstraZeneca and Cstone; grants and personal fees from Bayer, Eisai, BristolMyers Squibb, Roche/Genentech, Merck, Pfizer, and Eli Lilly and Company. M. Morimoto, K. Rau, H. Lim, and K. Liang have nothing to disclose. M. Ikeda is an Editorial Board Member of Liver Cancer and reports fees for honoraria, advisory board, \& research funding from Eli Lilly and Company, Bayer, Eisai, AstraZeneca, Chugai, Takeda, \& Novartis; honoraria and research funding from Bristol-Myers Squibb, MSD, \& EA Pharma; fees for advisory 
boards and research funding from Nihon Servier and Aslan; fees for research funding from Merck Serono, Yakult, Ono, J-Pharma, Pfizer, Chiome Bioscience, \& GlaxoSmithKline; honoraria fees from Taiho, Teijin Pharma, Astellas, Sumitomo Dainippon, Gilead, \& Otsuka. C. Yen has nothing to disclose. P. Galle reports grants from Bayer as well as personal fees from Bayer, BMS, AstraZeneca, Sirtex, MSD, Eisai, Ipsen, and Roche. J. Llovet reports grants and personal fees from Bayer Pharmaceuticals, Eisai, Boehringer Ingelheim, \& Ipsen, grants from Bristol-Myers Squibb, and personal fees from Eli Lilly and Company, Celsion, Merck, Genentech, Roche, Glycotest, Nucleix, Sirtex, Mina Alpha, AstraZeneca, Axis, \& Medscape. B. Daniele reports personal fees and nonfinancial support from Ipsen, Sanofi, \& Bayer as well as personal fees from Esiai, Eli Lilly and Company, AstraZeneca, MSD, Roche, \& Amgen. D. McIlwain is an employee and stockholder of Eli Lilly and Company. R. Yoshikawa is a full-time employee and shareholder of Eli Lilly and Company. K. Nakamura is an employee of Eli Lilly Japan KK. C. Wang is an employee and stockholder of Eli Lilly and Company. P. Abada is an employee of Eli Lilly and Company. R. Widau is an employee and stockholder of Eli Lilly and Company. A. Zhu is an Associate Editor of Liver Cancer and reports personal fees from Roche, Lilly, Eisai, Bayer, Merck, Exelixis, \& Sanofi.

\section{Funding Sources}

This study was funded by Eli Lilly and Company.

\section{Author Contributions}

All the authors approved the final version of the manuscript for submission to Liver Cancer and have participated sufficiently in the work to agree to be accountable for all aspects of the work in ensuring that questions related to the accuracy or integrity of any part of the work are appropriately investigated and resolved. Conception of the work: H. Lim, R. Yoshikawa, R. Widau, A. Zhu, M. Kudo, and R. Finn. Design of the work: H. Lim, R. Widau, A. Zhu, and M. Kudo. Acquisition of data for the work: M. Morimoto, M. Ikeda, P. Galle, H. Lim, A. Zhu, M. Kudo, and R. Finn. Analysis of data for the work: P. Galle, J. Llovet, H. Lim, K. Liang, C. Wang, P. Abada, R. Widau, and R. Finn. Interpretation of data for the work: K. Rau, M. Ikeda, C. Yen, P. Galle, J. Llovet, B. Daniele, H. Lim, D. McIlwain, R. Yoshikawa, K. Nakamura, C. Wang, P. Abada, R. Widau, A. Zhu, M. Kudo, and R. Finn. Drafting of the work: M. Morimoto, K. Rau, H. Lim, D. McIlwain, K. Nakamura, and R. Widau. Critical revision of the work for important intellectual content: M. Ikeda, C. Yen, P. Galle, J. Llovet, B. Daniele, H. Lim, R. Yoshikawa, K. Nakamura, K. Liang, C. Wang, P. Abada, R. Widau, A. Zhu, M. Kudo, R. Finn.

\section{References}

1 Llovet JM, Montal R, Sia D, Finn RS. Molecular therapies and precision medicine for hepatocellular carcinoma. Nat Rev Clin Oncol. 2018; 15:599-616

2 Bruix J, Reig M, Sherman M. Evidence-based diagnosis, staging, and treatment of patients with hepatocellular carcinoma. Gastroenterology. 2016;150:835-53.

3 Forner A, Reig M, Bruix J. Hepatocellular carcinoma. Lancet. 2018;391(10127):1301-14.

4 Kudo M. Heterogeneity and subclassification of Barcelona clinic liver cancer stage B. Liver Cancer. 2016;5:91-6.

5 Kudo M, Ueshima K, Chan S, Minami T, Chishina H, Aoki T, et al. Lenvatinib as an initial treatment in patients with intermediate-stage hepatocellular carcinoma beyond up-to-seven criteria and child-pugh a liver function: a proofof-concept study. Cancers. 2019;11:1084.

6 Di Costanzo GG, Tortora R. Intermediate hepatocellular carcinoma: how to choose the best treatment modality? World J Hepatol. 2015;7:1184-91.

7 Elshaarawy O, Gomaa A, Omar H, Rewisha E, Waked I. Intermediate stage hepatocellular carcinoma: a summary review. J Hepatocell Carcinoma. 2019;6:105-17.

8 Galle PR, Tovoli F, Foerster F, Wörns MA, Cucchetti A, Bolondi L. The treatment of intermediate stage tumours beyond TACE: from surgery to systemic therapy. J Hepatol. 2017;67:173-83.

9 Sun J-Y, Yin T, Zhang X-Y, Lu X-J. Therapeutic advances for patients with intermediate hepatocellular carcinoma. J Cell Physiol. 2019;234:12116-21.

10 Kudo M. Clinical practice guidelines for hepatocellular Carcinoma differ between Japan, United States, and Europe. Liver Cancer. 2015;4:85-95.

11 Park J-W, Chen M, Colombo M, Roberts LR, Schwartz M, Chen P-J, et al. Global patterns of hepatocellular carcinoma management from diagnosis to death: the BRIDGE Study. Liver Int. 2015;35:2155-66.

12 Kokudo N, Takemura N, Hasegawa K, Takayama T, Kubo S, Shimada M, et al. Clinical practice guidelines for hepatocellular carcinoma: the Japan society of hepatology 2017 (4th JSH-HCC guidelines) 2019 update. Hepatol Res. 2019;49:1109-13.

13 Forner A, Llovet JM, Bruix J. Hepatocellular carcinoma. Lancet. 2012;379:1245-55.

14 Llovet JM, Bruix J. Systematic review of randomized trials for unresectable hepatocellular carcinoma: Chemoembolization improves survival. Hepatology. 2003;37:429-42.

15 Raoul JL, Kudo M, Finn RS, Edeline J, Reig M, Galle PR. Systemic therapy for intermediate and advanced hepatocellular carcinoma: sorafenib and beyond. Cancer Treat Rev. 2018;68:16-24.

16 Llovet JM, Ricci S, Mazzaferro V, Hilgard P, Gane E, Blanc JF, et al. Sorafenib in advanced hepatocellular carcinoma. N Engl J Med. 2008:359:378-90.

17 Nishikawa H, Takeda H, Tsuchiya K, Joko K, Ogawa C, Taniguchi $\mathrm{H}$, et al. Sorafenib therapy for BCLC stage $\mathrm{B} / \mathrm{C}$ hepatocellular carci- noma; clinical outcome and safety in aged patients: a multicenter study in Japan. J Cancer. 2014;5:499-509.

18 Bruix J, Qin S, Merle P, Granito A, Huang YH, Bodoky G, et al. Regorafenib for patients with hepatocellular carcinoma who progressed on sorafenib treatment (RESORCE): a randomised, double-blind, placebo-controlled, phase 3 trial. Lancet. 2017;389:56-66.

19 Kudo M, Finn RS, Qin S, Han KH, Ikeda K, Piscaglia F, et al. Lenvatinib versus sorafenib in first-line treatment of patients with unresectable hepatocellular carcinoma: a randomised phase 3 non-inferiority trial. Lancet. 2018;391:1163-73.

20 Abou-Alfa GK, Meyer T, Cheng AL, ElKhoueiry AB, Rimassa L, Ryoo BY, et al. Cabozantinib in patients with advanced and progressing hepatocellular carcinoma. $\mathrm{N}$ Engl J Med. 2018;379:54-63.

21 El-Khoueiry AB, Sangro B, Yau T, Crocenzi TS, Kudo M, Hsu C, et al. Nivolumab in patients with advanced hepatocellular carcinoma (CheckMate 040): an open-label, noncomparative, phase $1 / 2$ dose escalation and expansion trial. Lancet. 2017;389:2492-502.

22 Kudo M. A new era in systemic therapy for hepatocellular carcinoma: atezolizumab plus bevacizumab combination therapy. Liver Cancer. 2020;9:119-37.

23 Finn RS, Qin S, Ikeda M, Galle PR, Ducreux M, Kim TY, et al. Atezolizumab plus bevacizumab in unresectable hepatocellular carcinoma. N Engl J Med. 2020;382:1894-905.
Ramucirumab for Intermediate-Stage

Hepatocellular Carcinoma
Liver Cancer 2021;10:451-460

DOI: $10.1159 / 000516605$ 
24 Finn RS, Ryoo BY, Merle P, Kudo M, Bouattour M, Lim HY, et al. Pembrolizumab as second-line therapy in patients with advanced hepatocellular carcinoma in KEYNOTE-240: a randomized, double-blind, phase III trial. J Clin Oncol. 2020;38:193-202.

25 Zhu AX, Kang YK, Yen CJ, Finn RS, Galle PR, Llovet JM, et al. Ramucirumab after sorafenib in patients with advanced hepatocellular carcinoma and increased $\alpha$-fetoprotein concentrations (REACH-2): a randomised, doubleblind, placebo-controlled, phase 3 trial. Lancet Oncol. 2019;20:282-96.

26 Zhu AX, Park JO, Ryoo B-Y, Yen C-J, Poon R, Pastorelli D, et al. Ramucirumab versus placebo as second-line treatment in patients with advanced hepatocellular carcinoma following first-line therapy with sorafenib (REACH): a randomised, double-blind, multicentre, phase 3 trial. Lancet Oncol. 2015;16:859-70.

27 Kudo M. Better efficacy of ramucirumab in Japanese patients than in the global population with unresectable hepatocellular carcinoma. Liver Cancer. 2020;9:232-44.

28 Kudo M, Galle PR, Brandi G, Kang Y-K, Yen C-J, Finn RS, et al. Effect of ramucirumab on ALBI grade in patients with advanced HCC: results from REACH and REACH-2. JHEP Reports. 2021;3(2):100215.

29 Johnson PJ, Berhane S, Kagebayashi C, Satomura S, Teng M, Reeves HL, et al. Assessment of liver function in patients with hepatocellular carcinoma: a new evidence-based approach-the ALBI grade. J Clin Oncol. 2015; 33:550-8.

30 Galle PR, Foerster F, Kudo M, Chan SL, Llovet JM, Qin S, et al. Biology and significance of alpha-fetoprotein in hepatocellular carcinoma. Liver Int. 2019;39:2214-29.

31 Kudo M. A new treatment option for intermediate-stage hepatocellular carcinoma with high tumor burden: Initial lenvatinib therapy with subsequent selective TACE. Liver Cancer. 2019;8:299-311.

32 Golfieri R, Bargellini I, Spreafico C, Trevisani F. Patients with barcelona clinic liver cancer stages $B$ and $C$ hepatocellular carcinoma: time for a subclassification. Liver Cancer. 2019;8: $78-91$.

33 Llovet JM, Villanueva A, Marrero JA, Schwartz M, Meyer T, Galle PR, et al. Trial design and endpoints in hepatocellular carcinoma: AASLD consensus conference. Hepatology. 2021;73(Suppl 1):158-91.
34 Piscaglia F, Ogasawara S. Patient selection for transarterial chemoembolization in hepatocellular carcinoma: importance of benefit/ risk assessment. Liver Cancer. 2018;7:104-19.

35 Llovet JM, Zucman-Rossi J, Pikarsky E, Sangro B, Schwartz M, Sherman M, et al. Hepatocellular carcinoma. Nat Rev Dis Primers. 2016;2:16018

36 Bolondi L, Burroughs A, Dufour JF, Galle PR, Mazzaferro V, Piscaglia F, et al. Heterogeneity of patients with intermediate (BCLC B) Hepatocellular Carcinoma: proposal for a subclassification to facilitate treatment decisions. Semin Liver Dis. 2012;32:348-59.

37 Kudo M, Arizumi T, Ueshima K, Sakurai T, Kitano M, Nishida N. Subclassification of BCLC B stage hepatocellular carcinoma and treatment strategies: proposal of modified bolondi's subclassification (kinki criteria). Dig Dis. 2015;33:751-8.

38 Kudo M, Han KH, Ye SL, Zhou J, Huang YH Lin SM, et al. A changing paradigm for the treatment of intermediate-stage hepatocellular carcinoma: Asia-Pacific primary liver cancer expert consensus statements. Liver Cancer. 2020;9:245-60.

39 Arizumi T, Minami T, Chishina $\mathrm{H}$, Kono $\mathrm{M}$, Takita M, Yada N, et al. Impact of tumor factors on survival in patients with hepatocellular carcinoma classified based on kinki criteria stage B2. Dig Dis. 2017;35:583-8.

40 Facciorusso A. Drug-eluting beads transarterial chemoembolization for hepatocellular carcinoma: current state of the art. World J Gastroenterol. 2018;24:161-9.

41 Guiu B, Deschamps F, Aho S, Munck F, Dromain C, Boige V, et al. Liver/biliary injuries following chemoembolisation of endocrine tumours and hepatocellular carcinoma: lipiodol vs. drug-eluting beads. J Hepatol. 2012;56:609-17.

42 Hiraoka A, Kumada T, Kudo M, Hirooka M, Koizumi Y, Hiasa Y, et al. Hepatic function during repeated TACE procedures and prognosis after introducing sorafenib in patients with unresectable hepatocellular carcinoma: multicenter analysis. Dig Dis. 2017;35:60210.

43 Sun Z, Li G, Ai X, Luo B, Wen Y, Zhao Z, et al. Hepatic and biliary damage after transarterial chemoembolization for malignant hepatic tumors: incidence, diagnosis, treatment, outcome and mechanism. Crit Rev Oncol Hematol. 2011;79:164-74.
$44 \mathrm{Tu}$ J, Jia Z, Ying X, Zhang D, Li S, Tian F, et al. The incidence and outcome of major complication following conventional TAE/TACE for hepatocellular carcinoma. Medicine. 2016;95:e5606.

45 Yau T, Kang YK, Kim TY, El-Khoueiry AB, Santoro A, Sangro B, et al. Efficacy and safety of nivolumab plus ipilimumab in patients with advanced hepatocellular carcinoma previously treated with sorafenib: the CheckMate 040 randomized clinical trial. JAMA Oncol. 2020;6:e204564.

46 Zhu AX, Finn RS, Edeline J, Cattan S, Ogasawara S, Palmer D, et al. Pembrolizumab in patients with advanced hepatocellular carcinoma previously treated with sorafenib (KEYNOTE-224): a non-randomised, openlabel phase 2 trial. Lancet Oncol. 2018;19: 940-52.

47 Yau T, Park JW, Finn RS, Cheng A-L, Mathurin P, Edeline J, et al. CheckMate 459: a randomized, multi-center phase III study of nivolumab (NIVO) vs sorafenib (SOR) as first-line (1L) treatment in patients (pts) with advanced hepatocellular carcinoma (aHCC). Ann Oncol. 2019;30:v874-5.

48 Abou-Alfa GK, Meyer T, Cheng AL, ElKhoueiry AB, Rimassa L, Ryoo BY, et al. Cabozantinib in patients with advanced and progressing hepatocellular carcinoma. N Engl J Med. 2018;379:54-63.

49 Bruix J, Qin S, Merle P, Granito A, Huang Y-H, Bodoky G, et al. Regorafenib for patients with hepatocellular carcinoma who progressed on sorafenib treatment (RESORCE): a randomised, double-blind, placebo-controlled, phase 3 trial. Lancet. 2017;389:56-66.

50 Finn RS, Ryoo B-Y, Merle P, Kudo M, Bouattour M, Lim HY, et al. Pembrolizumab as second-line therapy in patients with advanced hepatocellular carcinoma in KEYNOTE-240: a randomized, double-blind, phase III Trial. J Clin Oncol. 2019;38:193-202.

51 Finn RS, Qin S-K, Galle PR, Wang C, Ogburn $\mathrm{KD}$, Widau R, et al. 1015TiP Ramucirumab in patients with advanced hepatocellular carcinoma and elevated alpha fetoprotein following a non-sorafenib based systemic therapy: an expansion cohort of the phase III REACH-2 study. Ann Oncol. 2020;31:S702. 